\title{
MicroRNA-548ac induces apoptosis in laryngeal squamous cell carcinoma cells by targeting transmembrane protein 158
}

\author{
FUCUN SONG, YANG YANG and JIXIANG LIU \\ Department of Otolaryngology Head and Neck Surgery, Tianjin Union Medical Center, Tianjin 300121, P.R. China
}

Received December 30, 2019; Accepted June 18, 2020

DOI: 10.3892/ol.2020.11930

\begin{abstract}
Laryngeal cancer is a common head and neck cancer that effects the quality of life of those affected. Early diagnosis and treatment are vital to minimize the harmful effects of laryngeal cancer, which can improve the survival rate of patients following surgery and retain the voice function of the larynx. The purpose of the present study was to explore the molecular mechanism of the development of laryngeal cancer and to determine the biomarker for the diagnosis and treatment of laryngeal cancer. Reverse transcription-quantitative PCR (RT-qPCR) and The Cancer Genome Atlas database analysis were used to confirm high expression of TMEM158 in laryngeal cancer. The effects of TMEM158 and miR-548ac was investigated through in vitro and in vivo assays (MTT assay, colony-formation assay, flow cytometry assay, western blotting and tumor xenograft assay). Luciferase reporter assay, western blotting and RTq-PCR were used to confirm that miR-548 directly targeted the 3'-untranslated region of TMEM158 and inhibited TMEM158 expression. Taken together, the present results suggest that miR-548ac functions as a crucial cancer suppressor in laryngeal cancer, which induces apoptosis in laryngeal cancer cells by suppressing TMEM158. Thus, miR-548ac may be a potential target for the treatment of laryngeal cancer.
\end{abstract}

\section{Introduction}

Laryngeal cancer is a common primary malignant tumor in the head and neck (1). The incidence of laryngeal cancer is $5.7-7.6 \%$ of total body tumors (2). It is estimated that there were 177,422 cases of laryngeal cancer and 94,711 mortalities worldwide in 2012 (3). Currently, there is no exact cause of laryngeal cancer, which may be caused by several factors, including smoking, alcohol consumption, air pollution and viral infection $(4,5)$. Thus, the key to improving the prognosis of

Correspondence to: Dr Fucun Song, Department of Otolaryngology Head and Neck Surgery, Tianjin Union Medical Center, 190 Jieyuan Road, Tianjin 300121, P.R. China

E-mail: tjrmsong@126.com

Key words: transmembrane protein 158, microRNA-548ac, apoptosis, laryngeal cancer laryngeal cancer is to determine the biological characteristics of laryngeal cancer cells.

Transmembrane protein 158 (TMEM158), also known as RIS-1 protein, is recognized for its increased expression when Ras protein induces senescence of haploid fibroblasts infected with RAS V12 lentivirus (6). The expression and biological function of TMEM158 have been reported in different types of cancer (7-9); however, its role in laryngeal cancer remains unknown, thus, the present study aimed to investigate the regulatory mechanism and biological function of TMEM158 in laryngeal cancer.

MicroRNAs (miRNAs/miRs) are small non-coding RNA molecules that play key roles in the regulation of gene expression (10). Translation inhibition or silencing of target mRNAs is induced following complementary binding to the 3'-untranslated region (UTR) $(11,12)$. miR-548 is a large and poorly conserved primate-specific miRNA gene family that consists of 68 members, and several members of this family have been reported to be associated with cancer (13). For example, miR-548b-3p functions as a tumor suppressor in lung cancer (14) and as an antioncogenic regulator in breast cancer (15).

The purpose of the present study is to explore the molecular mechanism of the development of laryngeal cancer and to identify the biomarker for diagnosis and treatment of laryngeal cancer. Reverse transcription-quantitative PCR (RT-qPCR) and the analysis of The Cancer Genome Atlas (TCGA) database were used to demonstrate overexpression of TMEM158 in laryngeal cancer. The carcinogenic effect of TMEM158 and the anticancer effect of miR-548ac was investigated through in vitro and in vivo assays (MTT assay, colony formation assay, flow cytometry assay, western blotting and tumor xenograft assay). Luciferase reporter assay, western blotting and RT-qPCR were used to demonstrate miR-548 directly targeted the 3'-untranslated region of TMEM158 and inhibited TMEM158 expression. These results suggest that miR-548ac functions as a crucial cancer suppressor in laryngeal cancer, which induces apoptosis in laryngeal cancer cells by suppressing TMEM158. The present findings enrich the pathogenesis of laryngeal cancer and provide a new target and new ideas for the treatment on laryngeal cancer.

\section{Materials and methods}

Cell lines and culture. Human LSC-1 cells and NP69 nasopharyngeal epithelial cells were purchased from the Institute 
of Oncology, Chinese Academy of Medical Sciences. Cells were maintained in DMEM medium (Gibco; Thermo Fisher Scientific, Inc.) supplemented with $10 \%$ fetal bovine serum (cat. no. 10100147; Thermo Fisher Scientific, Inc.), penicillin $(100 \mathrm{IU} / \mathrm{ml})$ and streptomycin $(100 \mathrm{mg} / \mathrm{ml})$ at $37^{\circ} \mathrm{C}$ in $5 \% \mathrm{CO}_{2}$. LSC-1 cells were transfected with $10 \mu \mathrm{M}$ miR-548ac agomir (LSC-1-miR-548ac) or $10 \mu \mathrm{M}$ miR-548ac agomir negative control (NC; LSC-1-NC; Guangzhou RiboBio Co., Ltd.) using Lipofectamine ${ }^{\circledR}$ RNAiMAX reagent (cat. no. 13778030; Thermo Fisher Scientific, Inc.). The short hairpin (sh)RNA targeting positions 1520-1540 (5'-AAATGACCAAATCCT GTGTAT-3'; Sangon Biotech Co. Ltd.) of human TMEM158 mRNA was cloned into a lentiviral vector (PLKO.1). A non-specific scrambled shRNA sequence (5'-CCTAAGGTT AAGTCGCCCTCG-3'; Sangon Biotech Co. Ltd.) was used as the NC. $1 \mu \mathrm{g}(50 \mathrm{pmol})$ shRNA was added into Opti-MEM (cat. no. 31985062; Thermo Fisher Scientific, Inc.) to prepare RNA diluent, and the final volume was $25 \mu$ l. LSC- 1 cells were subsequently transfected with lentiviral packaging vectors (LSC-1-NC or LSC-1-shTMEM158) using Lipofectamine ${ }^{\circledR}$ 3000 reagent (cat. no. L3000001; Thermo Fisher Scientific, Inc.), according to the manufacturer's instructions. Subsequent experiments began at $24 \mathrm{~h}$ after transfection of LSC-1 cells with shRNA. The LSC-1 cells stably transfected with NC, miR-548ac and shTMEM158 were constructed respectively.

Tumor xenograft model. A total of 304 -week-old BALB/cA female nude mice (14-18 g) were purchased from the Beijing Vital River Laboratory Animal Technologies Co. Ltd. The present study was approved by the Ethics Committee of Tianjin Union Medical Center (Tianjin, China; approval no. 2018-269). All nude mice were kept at $28^{\circ} \mathrm{C}$ and SPF level with light/dark cycle. Mice received pathogen-free food and water. All food and water were sterilized and then sent into the animal isolator through a sterile entrance chamber to ensure that the nude mice were in a sterile living state. All animal experiments were performed in accordance with the National Institutes of Health Guide for the Care and Use of Laboratory Animals (NIH Publications no. 8023, revised in 1978).

LSC-1 cells $\left(2 \times 10^{6}\right.$ cells/mouse) were inoculated into the back of the nude mice following light anesthesia with $350 \mathrm{mg} / \mathrm{kg}$ chloral hydrate. The mice were randomly divided into four groups (6 mice/group), as follows: i) LSC-1 cells with a blank vector; ii) LSC-1 cells with shTMEM158; ii) LSC-1 cells with miR-548ac NC and iv) LSC-1 cells with miR-548ac agomir. The experimental procedures lasted for 3 weeks. The health and behavior of all animals were monitored every day and the individuals with poor health were terminated in time. The nude mice were free from stress, discomfort, pain and injury. All mice were sacrificed via intraperitoneal injection with $200 \mathrm{mg} / \mathrm{kg}$ pentobarbital sodium. Mortality was confirmed by checking for a heartbeat and examining the pupil dilation of the nude mice. The maximum diameter of the tumor was $0.87 \mathrm{~cm}$. The tumors were subsequently removed using resection, photographed using a camera (model, D3600; Nikon Corporation) and weighed. Tumor volume was calculated using the following formula: $\mathrm{V}=\left(\right.$ length $\mathrm{x}$ width $\left.{ }^{2}\right) / 2$.

MTT assay. The cell suspensions (LSC-1-NC, LSC-1miR-548ac; LSC-1-NC or LSC-1-shTMEM158) were added into 96-well plates at a density of 6,000 cells/well. After $24 \mathrm{~h}$ of cell attachment, the culture medium was discarded. Cells were subsequently incubated with serum-free DMEM $(100 \mu \mathrm{l})$ and $10 \mu 1 \mathrm{MTT}(5 \mathrm{mg} / \mathrm{ml}$; cat. no. ST316; Beyotime Institute of Biotechnology, Inc.) for $4 \mathrm{~h}$ at $37^{\circ} \mathrm{C}$. Following the MTT incubation, the purple formazan crystals were dissolved using $100 \mu \mathrm{l}$ dimethyl sulfoxide and shaking vigorously for $10 \mathrm{~min}$ using a vortex oscillator (model, MixMax; AIBENSEN, Co. Ltd.). Cell viability was subsequently analyzed at a wavelength of $570 \mathrm{~nm}$. All experiments were performed in triplicate and three biological repeats were conducted.

Colony formation assay. The cell suspensions (LSC-1-NC, LSC-1-miR-548ac; LSC-1-NC or LSC-1-shTMEM158) were added into 6-well plates at a density of 300 cells/well. Cells were maintained in DMEM medium supplemented with $10 \%$ fetal bovine serum (cat. no. 10100147; Thermo Fisher Scientific, Inc.) for 7-10 days at $37^{\circ} \mathrm{C}$. After $\sim 70$ cells appeared in each colony, each pore was fixed with $1 \mathrm{ml}$ methanol for $15 \mathrm{~min}$ at room temperature. Cell colonies were subsequently stained with $0.5 \%$ crystal violet for $10 \mathrm{~min}$ at room temperature, and excess stain was discarded using ultrapure water. Cell colonies were observed and recorded using a camera (model, D3600; Nikon Corporation).

Annexin V-propidium iodide (PI) assay. Cells (LSC-1-NC, LSC-1-miR-548ac; LSC-1-NC or LSC-1-shTMEM158) were digested with trypsin-EDTA solution (0.05\% trypsin and $0.02 \%$ EDTA; cat. no. C0202; Beyotime Institute of Biotechnology, Inc.) and collected by centrifugation at $161 \mathrm{x} \mathrm{g}$ and room temperature. Cells were washed three times with sterile PBS and subsequently resuspended in $500 \mu 1$ Annexin V-PI binding solution. PI (10 $\mu \mathrm{l})$ and annexin V-fluorescein isothiocyanate (5 $\mu \mathrm{l})$ (cat. no. C1062S; Beyotime Institute of Biotechnology, Inc.) were added to the cell suspensions of each group and manually gently mixed. The whole reaction was $515 \mu 1$ at room temperature and dark for $30 \mathrm{~min}$. Apoptotic cells were subsequently detected using a flow cytometer.

Flow cytometric analysis of cell cycle distribution. Cells (LSC-1-NC, LSC-1-miR-548ac; LSC-1-NC or LSC-1-shTMEM158) at $1 \times 10^{6}$ cells $/ \mathrm{ml}$ were collected following digestion with trypsin-EDTA solution (cat. no. C0202; Beyotime, Inc.), washed twice with PBS, and resuspended and fixed with $70 \%$ ethanol overnight at $-20^{\circ} \mathrm{C}$. The cell suspension was re-washed twice with PBS and stained with $400 \mu \mathrm{l}$ PI at $4^{\circ} \mathrm{C}$ for $30 \mathrm{~min}$ in the dark. Cell cycle distribution was subsequently detected via flow cytometric analysis.

Western blotting. Total protein from cells (LSC-1-NC, LSC-1-miR-548ac; LSC-1-NC or LSC-1-shTMEM158) was extracted using RIPA lysis buffer (cat. no. R0010; Beijing Solarbio Science \& Technology Co., Ltd.). Detection of protein concentration was used BCA assay. Equal amounts $(15 \mu \mathrm{g})$ of protein were separated via SDS-PAGE (12\% separating gel and 5\% concentrating gel) and subsequently transferred onto polyvinylidene difluoride (PVDF) membranes. The PVDF membrane was blocked in 5\% skimmed milk at room temperature for $2 \mathrm{~h}$. The membranes were incubated with primary antibodies against: Bax (1:1,000; cat. no. ab32503), 
B-cell lymphoma-2 (Bcl-2; 1:1,000; cat. no. ab8227), TMEM158 (1:1,000; cat. no. ab98335) and $\beta$-actin $(1: 2,000$; cat. no. ab98335) (all purchased from Abcam) at room temperature for $2 \mathrm{~h}$. Following the primary incubation, membranes were incubated with secondary antibodies (HRP goat anti-Rabbit; 1:5,000; cat. no. A0208; Beyotime Institute of Biotechnology, Inc.) at room temperature for $1 \mathrm{~h}$. Protein bands were visualized using enhanced chemiluminescence buffer and detected using the multifunctional gel imaging system (model, Gel Doc XR+; Bio-Rad Laboratories, Inc.). Protein expression was calculated via the gray value.

Reverse transcription-quantitative (RT-q)PCR. Total RNA was extracted from cells (LSC-1-NC, LSC-1-miR-548ac; LSC-1-NC or LSC-1-shTMEM158) using TRIzol ${ }^{\circledR}$ reagent (cat. no. 5301100; SIMGEN) according to the manufacturer's instructions, and quantified using a NanoDrop 2000 spectrophotometer (Thermo Fisher Scientific, Inc.). Total RNA was reverse transcribed into cDNA using the TransScript RT Reagent kit (cat. no. AH411-02; TransGen Biotech Co., Ltd.). The reverse transcription reaction conditions were $25^{\circ} \mathrm{C}, 10 \mathrm{~min}$ (miRNA skip); $42^{\circ} \mathrm{C}, 30 \mathrm{~min} ; 85^{\circ} \mathrm{C}, 5 \mathrm{~min}$. qPCR was subsequently performed using the $2 x S Y B R$ Green qPCR mix (cat. no. 153301; Beijing BLKW Biotechnology Co. Ltd.) and a ABI 7500 system (Thermo Fisher Scientific, Inc.). The qPCR reaction conditions were 1 cycle at $94^{\circ} \mathrm{C}$ for $2 \mathrm{~min} ; 40$ cycles $\left(94^{\circ} \mathrm{C}\right.$ for $15 \mathrm{sec} ; 55^{\circ} \mathrm{C}$ for $15 \mathrm{sec}$; and $72^{\circ} \mathrm{C}$ for $20 \mathrm{sec}$ ). The following primer sequences were used for qPCR: TMEM158 forward, 5'-TGCCCAACGGCATGG AACA-3' and reverse, 5'-AGGAGCGGAGCGGGTCACTT-3'; GAPDH forward, 5'-ATGACATCAAGAAGGTGGTGAAGC AGG-3' and reverse, GCGTCAAAGGTGGAGGAGTGGGT; miR-548ac forward, 5'-CAAAAACCGGCAATTACTTTT G-3' and reverse, 5'-CTCAACTGGTGTCGTGGA-3'; and U6 forward, 5'-CTCGCTTCGGCAGCACA-3' andreverse, 5'-CTCAACTGGTGTCGTGGA-3'. Relative expression levels were calculated using the $2^{-\Delta \Delta C q}$ method (16) and normalized to the internal reference genes GAPDH and U6, respectively.

Dual-luciferase reporter assay. The wild (WT)/mutant (MUT) 3'-UTR fragments of TMEME158 were amplified via PCR using 2X PCR Master mix (cat. no. D7228; Beyotime Institute of Biotechnology, Inc.). The PCR reaction conditions were: 1 cycle at $94^{\circ} \mathrm{C}$ for $5 \mathrm{~min} ; 35$ cycles $\left(94^{\circ} \mathrm{C}\right.$ for $30 \mathrm{sec}$; $60^{\circ} \mathrm{C}$ for $1 \mathrm{~min} ; 72^{\circ} \mathrm{C}$ at $\left.1 \mathrm{~min}\right)$; and 1 cycle $\left(72^{\circ} \mathrm{Cfor} 10 \mathrm{~min}\right)$. Th fragments were cloned into the Dual-Luciferase miRNA targeted expression vector (Promega Corporation) to synthesize WT/MUT-TMEM158-3'-UTR. LSC-1 cells with 70\% confluence were transfected with WT-TMEM158-3'-UTR or MUT-TMEM158-3'-UTR and miR-548ac agomirs or miR-548ac agomirs NC/miR-548ac antagonists or miR-548ac antagonists NC(Guangzhou RiboBio Co., Ltd.) usingLipofectamine ${ }^{\circledR} 3000$ reagent (cat. no. L3000008; Thermo Fisher Scientific, Inc.). Following incubation at $37^{\circ} \mathrm{Cfor} 48 \mathrm{~h}$, Luciferase activity was measured using a multimode microplate reader (BioTek Instruments, Inc.) and normalized with Renilla luciferase activity.

Bioinformatics analysis. The mRNA and miRNA sequencing data and the corresponding clinical information of the patients with laryngeal cancer were downloaded from the TCGA data portal (http://cancergenome.nih.gov). The data were screened and laryngeal cancer samples and non-tumor samples, which had detailed clinical information (laryngeal cancer, 116 cases; normal, 11 cases), were selected. The clinical features of the patients are shown in Table I. Patients with head and neck cancer were divided into four stages, according to tumor-node-metastasis (TNM) (17) and differences in TMEM158 expression at different Tumor-Node-Metastasis stages were analyzed. RNAInter software (http://www. rna-society.org/raid/; Department of Bioinformatics, Southern Medical University) was used to predict the target association between miR-548ac and TMEM158. Univariate Cox proportional hazards regression analysis was performed to determine the association between TMEM158 expression and overall survival rate. Kaplan-Meier survival analysis and log-rank test were used to assess the association between TMEM158 expression and overall survival time. A total of 38 cases of TMEM158 with high or low expression were selected.

Statistical analysis. Statistical analyses were performed using SPSS 18.0 software (SPSS, Inc.). All experiments were performed in triplicate and data are presented as the mean \pm standard deviation. Unpaired Student's two-tailed t-test was used to compare differences between two independent samples, whilst one-way analysis of variance and Bonferroni's correction were used to compare differences between multiple groups (Figs. 1B, 2F, 3G, 4D and 5B and C). $\mathrm{P}<0.05$ was considered to indicate a statistically significant difference.

\section{Results}

TMEM158 is upregulated in laryngeal cancer tissues and cell lines. The Cancer Genome Atlas (TCGA) database was used to compare mRNA sequencing data between laryngeal carcinoma tissues and adjacent normal tissues. The results demonstrated that TMEM158 expression was significantly higher in laryngeal carcinoma tissues compared with adjacent normal tissues (Fig. 1A). TMEM158 expression according to different tumor-node-metastasis (TNM) stages was also assessed. The results demonstrated that TMEM158 expression increased in accordance with the increase of the TNM stage (Fig. 1B). The prognostic value of TMEM158 in laryngeal carcinoma was determined via overall survival analysis. The results demonstrated that patients with low TMEM158 expression had a significantly longer survival time than that of patients with high TMEM158 expression (Fig. 1C). TMEM158 mRNA expression was further assessed in NP69 and LSC-1 cells. The results demonstrated that TMEM158mRNA expression was $4.35 \pm 0.56$ times greater in LSC-1 cells compared with NP69 cells $(\mathrm{P}=0.0052$; Fig. 1D). Taken together, these results suggest that upregulated TMEM158 expression may play a critical role in the development and progression of laryngeal cancer.

Effect of TMEM158 on the viability of laryngeal cancer cells. Normal LSC-1 cells were used as the control group, while cells transfected with sh-TMEM158 were used as the experimental group. RT-qPCR analysis demonstrated that TMEM158 mRNA expression was significantly lower in LSC-1 cells transfected with sh-TMEM158 compared with LSC-1 cells 
A

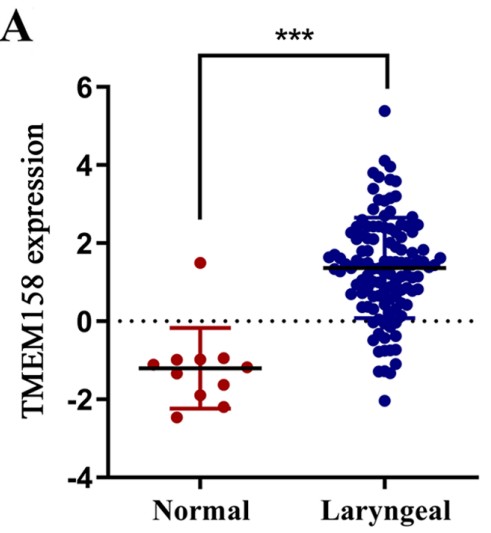

C

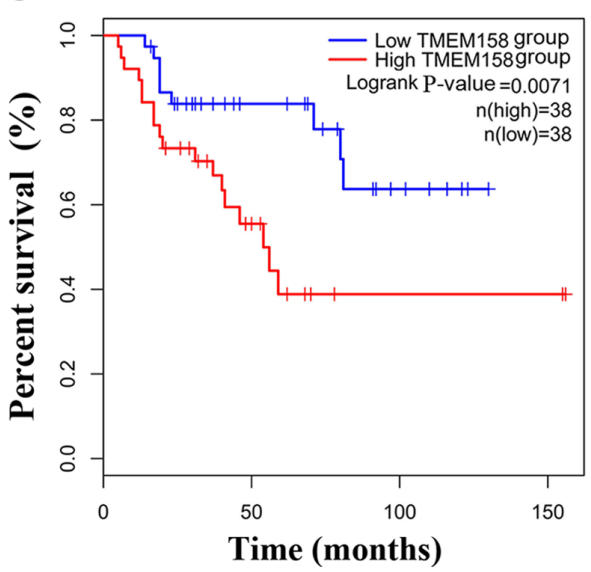

B

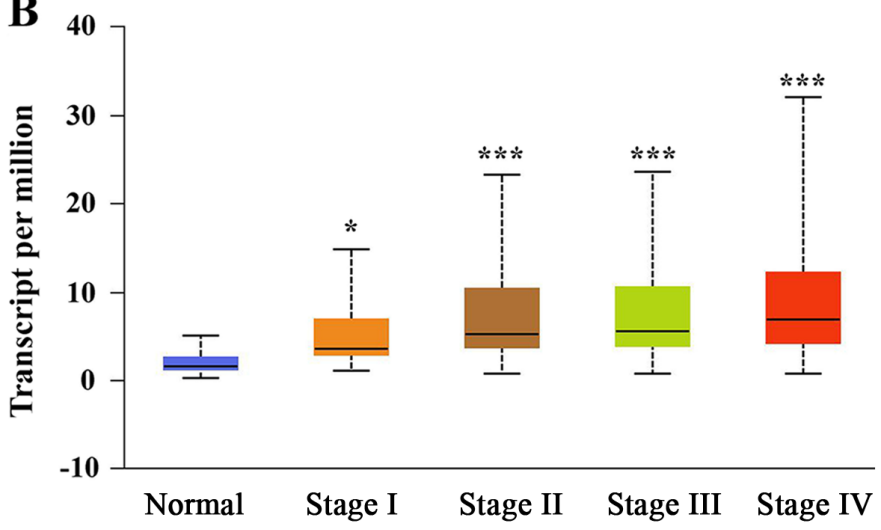

D

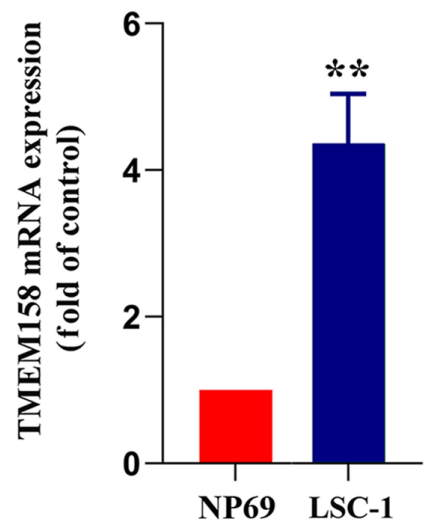

Figure 1. TMEM158 expression using data downloaded from TCGA database. (A) TMEM158 expression was assessed in laryngeal carcinoma tissues and adjacent normal tissues using mRNA sequencing data from the TCGA database. Red represents cancer tissues and blue represents adjacent normal tissues. ${ }^{* * *} \mathrm{P}<0.001$, normal tissue vs. laryngeal cancer. (B) TMEM158 expression at different tumor-node-metastasis stages. ${ }^{*} \mathrm{P}<0.05$ and ${ }^{* * *} \mathrm{P}<0.001$, stage I/II/III/IV vs. normal. (C) Kaplan-Meier survival analysis of patients with laryngeal cancer based on TMEM158 expression. ${ }^{* * *} \mathrm{P}<0.001$, lowTMEM158 group vs. highTMEM158 group. (D) Reverse transcription-quantitative PCR was performed to assess TMEM158 mRNA expression in human laryngeal squamous cell carcinoma cells and NP69 nasopharyngeal epithelial cells. ${ }^{* *} \mathrm{P}<0.01$, LSC-1 cells vs. NP69 cells. TMEM158, transmembrane protein 158; TCGA, The Cancer Genome Atlas.

transfected with $\mathrm{NC}(14.67 \pm 2.07 \%$ of the control; $\mathrm{P}<0.001$; Fig. 2A). The results of western blot analysis were consistent with these findings (Fig. 2B). Similarly, the results of the MTT assay demonstrated that cell viability was significantly lower in LSC-1 cells transfected with sh-TMEM158 compared with the control group $(51.49 \pm 4.51 \%$ of the control; $\mathrm{P}<0.0001$; Fig. 2C). The results of the colony formation assay demonstrated that the number of cell colonies was significantly lower in the experimental group compared with the control group $(67.62 \pm 4.72 \%$ of the control; $\mathrm{P}=0.025$; Fig. 2D). The results of the Annexin V-PI assay indicated that the apoptotic rate was significantly higher in the experimental group compared with the control group $(7.3 \pm 1.33$ of the control; $\mathrm{P}=0.00062$; Fig. 2E). The cell cycle distribution assay demonstrated a higher proportion of cells transfected with sh-TMEM158 in the $G_{1}$ phase compared with the control group (Fig. 2F). The protein expression levels of $\mathrm{Bax}$ and $\mathrm{Bcl}-2$ were determined via western blot analysis, and the gray value was used for relative quantification. The results demonstrated that Bax and $\mathrm{Bcl}-2$ expression levels were significantly higher in the experimental group compared with the control group $(4.53 \pm 0.03$ of the control; P=0.00072; Fig. 2G). Collectively, these results indicate that silencing TMEM158 significantly inhibits the viability of laryngeal cancer cells.

miR-548ac is downregulated in laryngeal cancer tissues and cell lines. RNAInter software analysis demonstrated that miR-548ac has a high probability of binding to TMEM158. TCGA database was used to compare miRNA sequencing data between laryngeal carcinoma tissues and adjacent normal tissues. The results demonstrated that miR-548ac expression was significantly lower in laryngeal carcinoma tissues compared with adjacent normal tissues (Fig. 3A). miR-548ac expression was further assessed inNP69 and LSC-1 cells. The results demonstrated that miR-548ac expression was significantly lower in LSC-1 cells compared with NP69 cells $(68.67 \pm 10.21 \%$ of NP69 cells; P=0.027; Fig. 3B). Taken together, these results suggest that downregulation of miR-548ac may play a critical role in the progression of laryngeal cancer.

Association between miR-548ac and TMEM158. miR-548ac agomir (experimental group) or miR-548ac agomir NC (control group) were transfected into LSC-1 cells, respectively. RT-qPCR analysis demonstrated that miR-548ac expression 
A
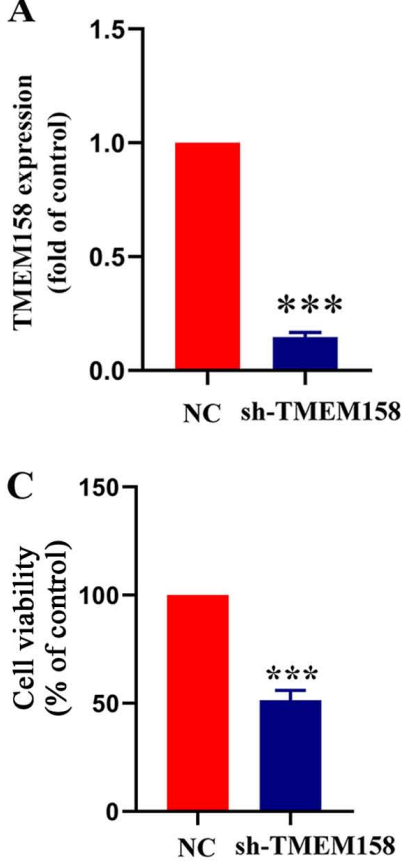

$\mathbf{E}$

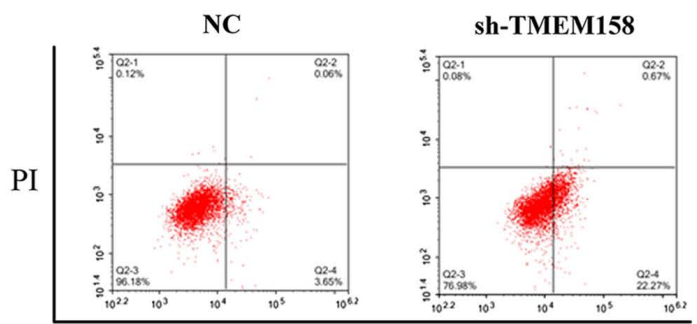

Annexin V

F

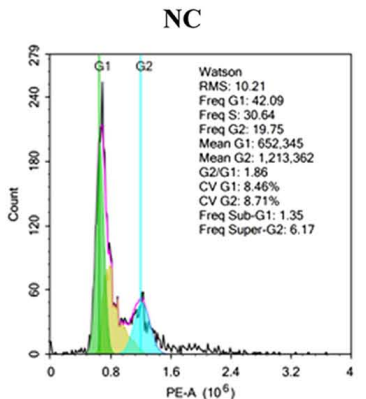

B

\section{NC sh-TMEM158}

D
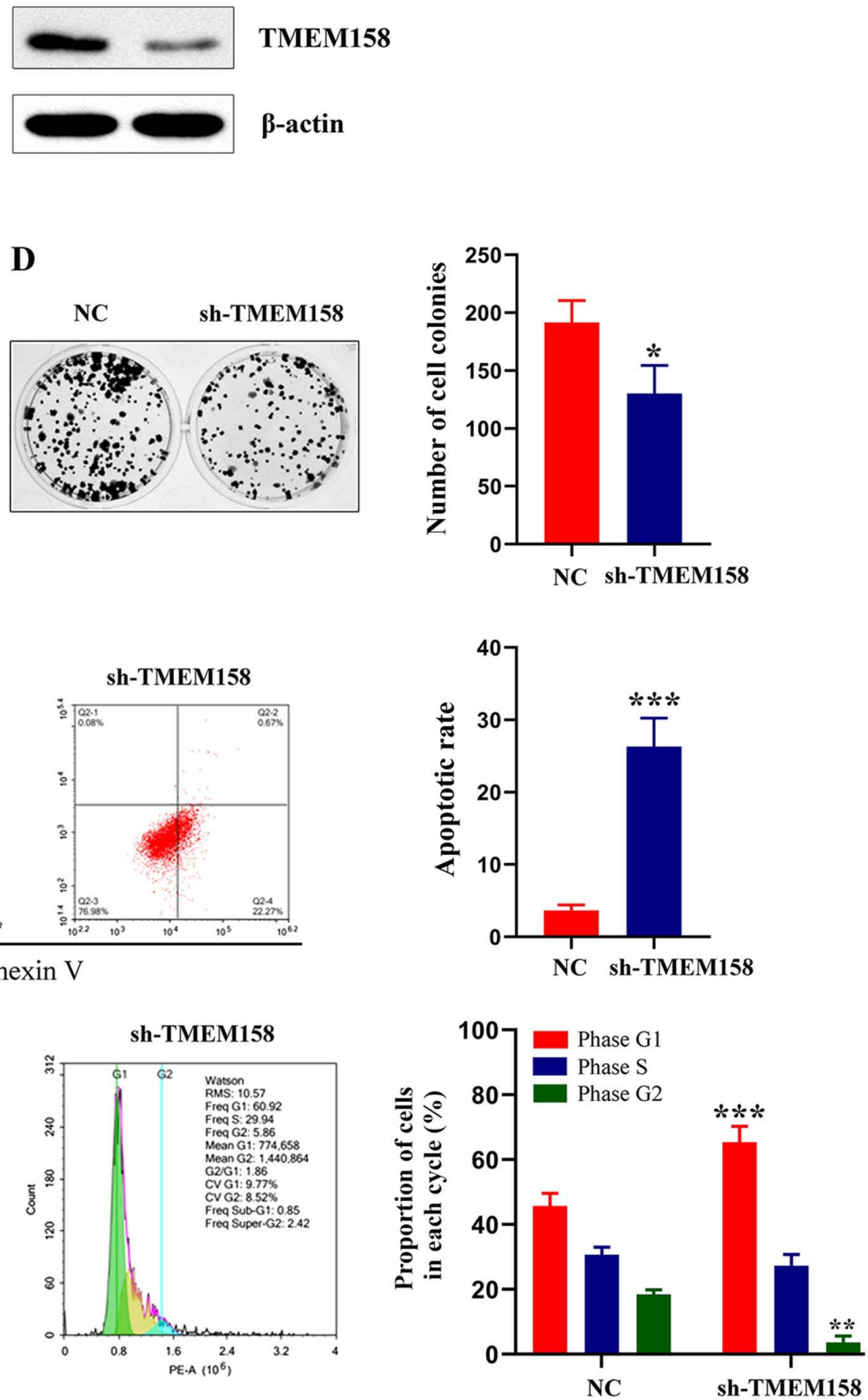

G

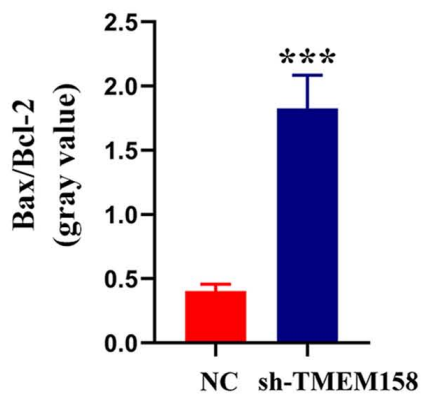

Figure 2. TMEM158 affects viability, formation of colonies, cell cycle distribution and apoptosis of laryngeal cancer cells. (A) Reverse transcription-quantitative PCR and (B) Western blot analyses were performed to assess mRNA and protein expression of TMEM158 in LSC-1 cells transfected with sh-TMEM158 or NC, respectively. ${ }^{* * *} \mathrm{P}<0.001$, shTMEM158 vs. NC. (C) The MTT assay was performed to assess cell viability in LSC-1 cells transfected with sh-TMEM158 or NC. ${ }^{* * *} \mathrm{P}<0.001$, shTMEM158 vs. NC. (D) Effect of TMEM158 on colony formation of laryngeal cancer cells. Representative photos (left) and relative quantification analysis of colonies (right). ${ }^{*} \mathrm{P}<0.05$, shTMEM158 vs. NC. (E) Flow cytometric analysis of apoptosis. ${ }^{* * *} \mathrm{P}<0.001$, shTMEM158 vs. NC. (F) Flow cytometric analysis of cell cycle distribution. Histograms depict the proportion of LSC-1 cells in the G1, S and G2 phases. ${ }^{* * *} \mathrm{P}<0.001$, shTMEM158 (G $\left.\mathrm{G}_{1}\right)$ vs. NC $\left(\mathrm{G}_{1}\right) ;{ }^{* * *} \mathrm{P}<0.01$, shTMEM158 $\left(\mathrm{G}_{2}\right)$ vs. NC $\left(\mathrm{G}_{2}\right)$. $(\mathrm{G})$ Western blot analysis was performed to assess protein expression levels of Bax and Bcl-2. Histograms depict the gray value of Bax/Bcl-2. ${ }^{* * *} \mathrm{P}<0.001$, shTMEM158 vs. NC. TMEM158, transmembrane protein 158; LSC-1, laryngeal squamous cell carcinoma; sh, short hairpin; NC, negative control; Bcl-2, B-cell lymphoma 2; PI, propidium iodide. 

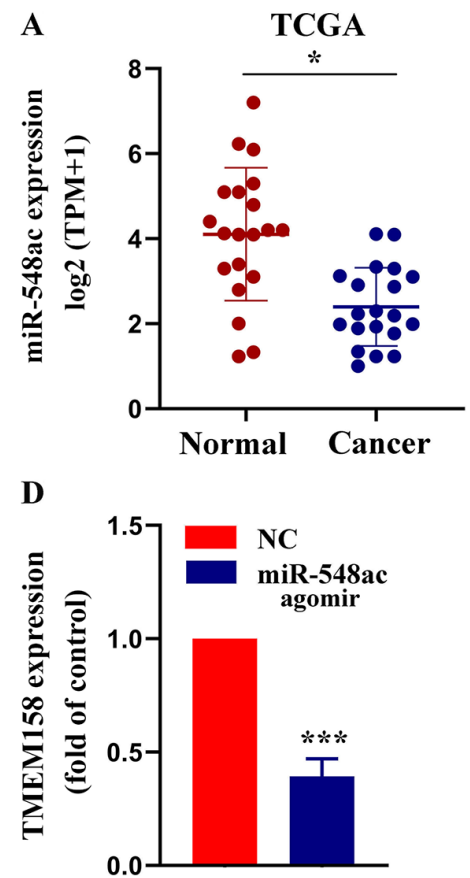

F

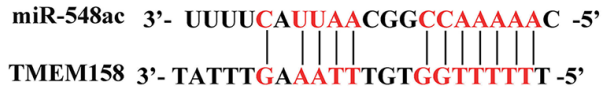

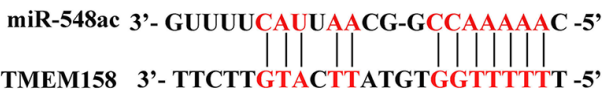

B

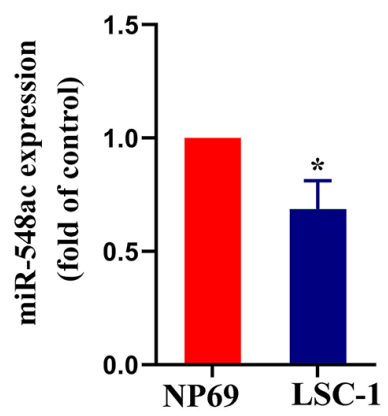

$\mathbf{E}$

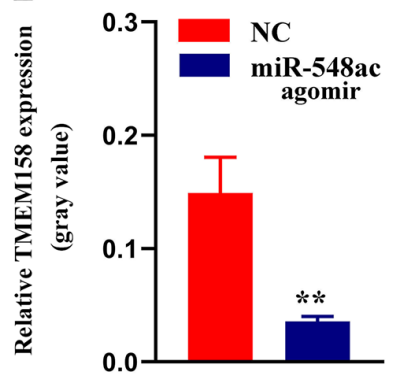

G

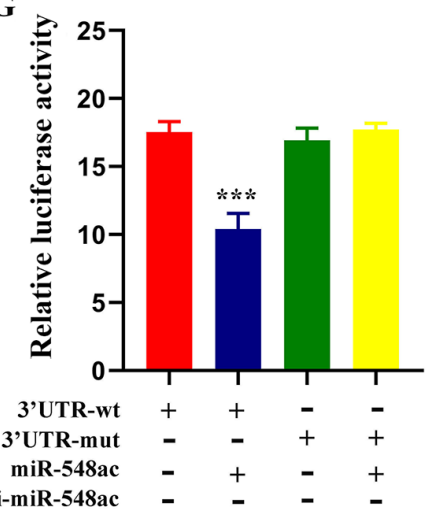

C

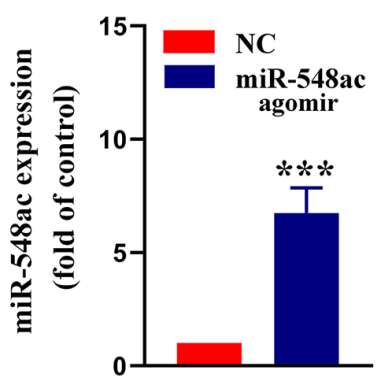

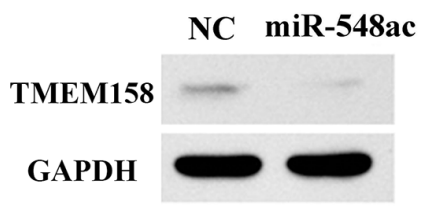

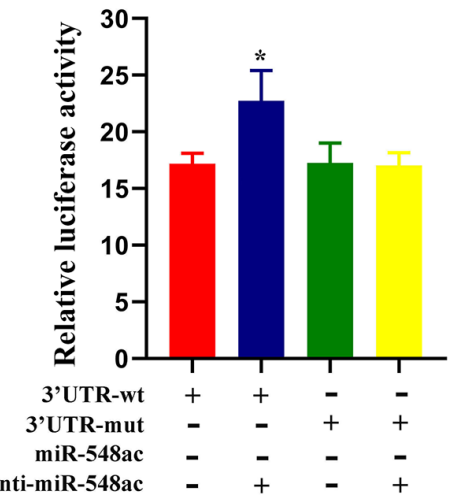

Figure 3. miR-548ac suppresses TMEM158 expression by directly binding to its 3'-UTR. (A) miR-548ac expression was assessed in laryngeal carcinoma tissues and adjacent normal tissues using miRNA sequencing data from TCGA database. * P<0.05 normal group vs. tumor group. (B) RT-qPCR analysis was performed to assess miR-548ac expression in NP69 and LSC-1 cells. ${ }^{*} \mathrm{P}<0.05$, LSC-1 cells vs. NP69 cells. (C) RT-qPCR assay was performed to assessmiR-548ac expression in LSC-1 cells transfected with miR-548ac agomir or NC. ${ }^{* * * *} \mathrm{P}<0.001$, miR-548ac agomirs vs. NC. (D) RT-qPCR analysis was performed to assess TMEM158 expression in LSC-1 cells transfected with miR-548ac agomir or NC. ${ }^{* * *} \mathrm{P}<0.001$, miR-548ac agomirs vs. NC. (E) Western blot analysis was performed to assess TMEM158 protein expression in LSC-1 cells transfected with miR-548ac agomir or NC. Histograms depict the gray value of TMEM158/GAPDH. ** P<0.01, miR-548ac agomirs vs. NC. (F) Sequence alignment of miR-548ac and its predicted binding sites (red) in TMEM158 3'-UTR. (G) Luciferase reporter assay. ${ }^{* * *}$ P<0.001, 3'UTR-WT (+miR-548ac) vs. 3'UTR-WT; ${ }^{*} \mathrm{P}<0.05,3^{\prime} \mathrm{UTR}-\mathrm{WT}$ (+Anti-miR-548ac) vs. 3'UTR-WT. miR, microRNA; TMEM158, transmembrane protein 158; UTR, untranslated region; TCGA, The Cancer Genome Atlas; RT-qPCR, reverse transcription-quantitative PCR; LSC-1, laryngeal squamous cell carcinoma; NC, negative control.

was significantly higher in the experimental group compared with the control group (7.76 \pm 1.1 -fold of the control; $\mathrm{P}<0.001$; Fig. 3C). Furthermore, TMEM158 mRNA expression was significantly lower in the experimental group compared with the control group $(39.33 \pm 6.34 \%$ of the control; $\mathrm{P}=0.00071$; Fig. 3D). The results of western blot analysis were consistent with these findings (Fig. 3E). The results of the dual-luciferase reporter assay demonstrated that luciferase activity decreased in LSC-1 cells transfected with WT-TMEM158-3'-UTR and miR-548ac agomir compared with WT-TMEM158-3'-UTR alone $(59.58 \pm 6.78 \%$ of WT-TMEM158-3'-UTR alone; $\mathrm{P}<0.001)$. However, the luciferase activity remained unchanged whenLSC-1 cells were transfected with MUT-TMEM158-3'-UTR and miR-548ac agomir. Luciferase activity increased whenLSC-1 cells were transfected
withWT-TMEM158-3'-UTR and anti-miR-548ac agomir compared with WT-TMEM158-3'-UTR alone (1.32 \pm 0.07 of WT-TMEM158-3'-UTR alone; $\mathrm{P}=0.03$ ). However, the luciferase activity remained unchanged whenLSC-1 cells were transfected with MUT-TMEM158-3'-UTR and anti-miR-548ac agomir compared with MUT-TMEM158-3'-UTR alone (Fig. 3G). Collectively, these results indicate that TMEM158 is a direct target gene of miR-548ac.

Effect of miR-548ac on the viability of laryngeal cancer cells. Normal LSC-1 cells were used as the control group, while cells transfected with miR-548ac agomir were used as the experimental group. The results of the MTT assay demonstrated that cell viability significantly decreased in the experimental group compared with the control group $(51.86 \pm 3.11 \%$ of the control; 


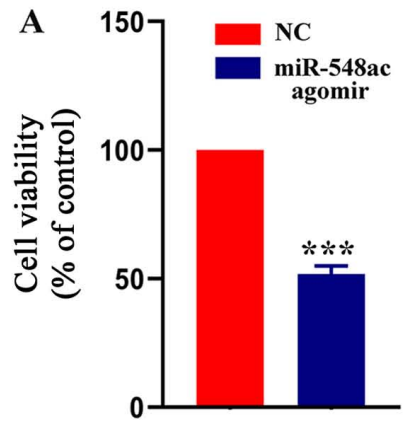

B

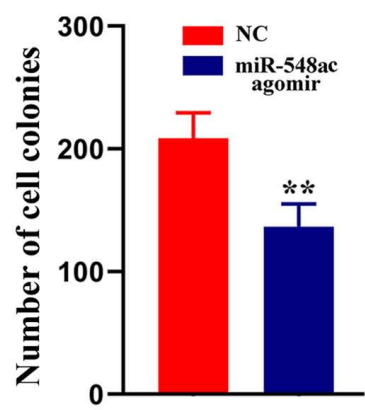

$\mathbf{C}$
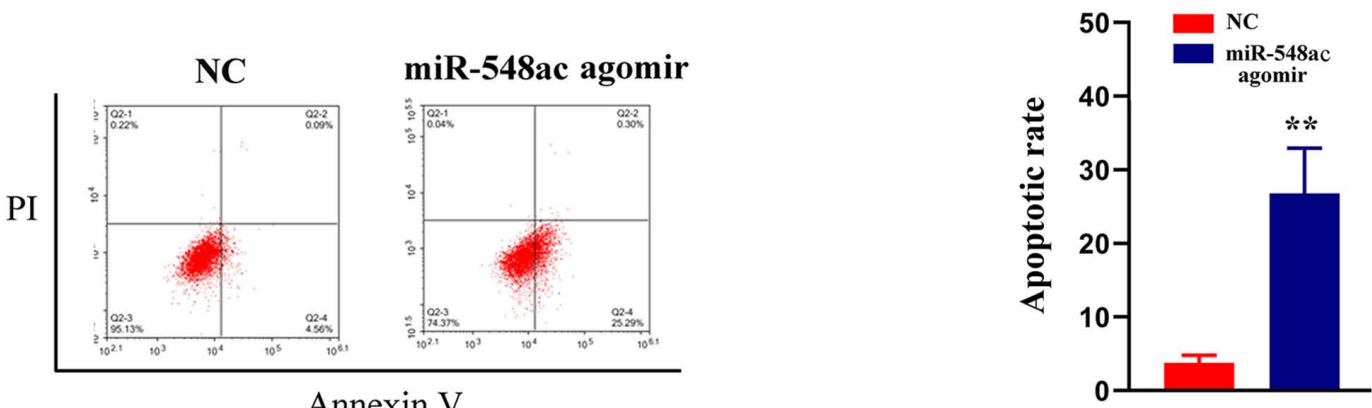

D
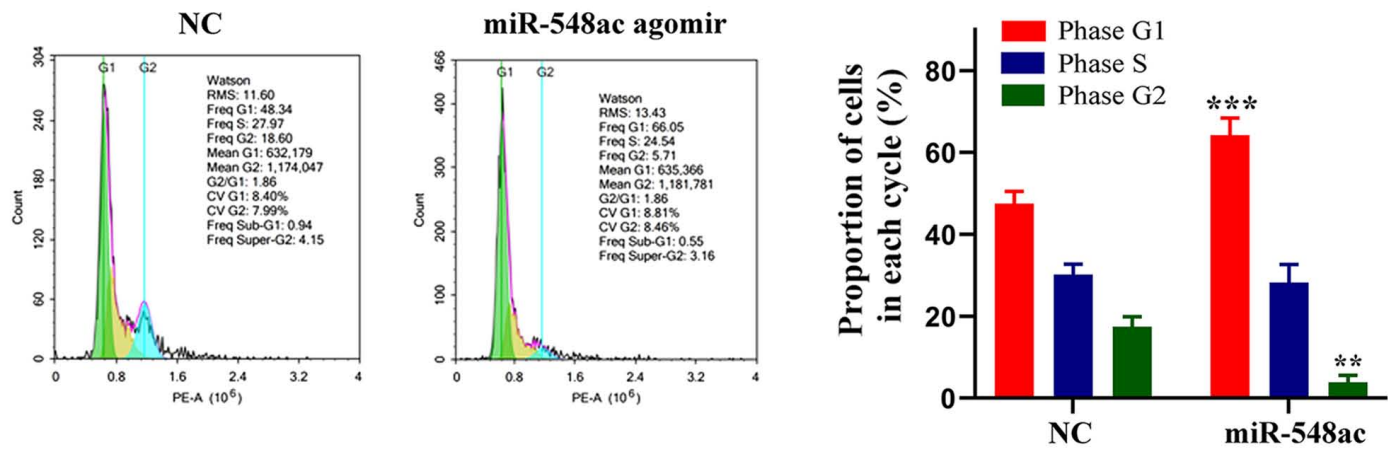

$\mathbf{E}$
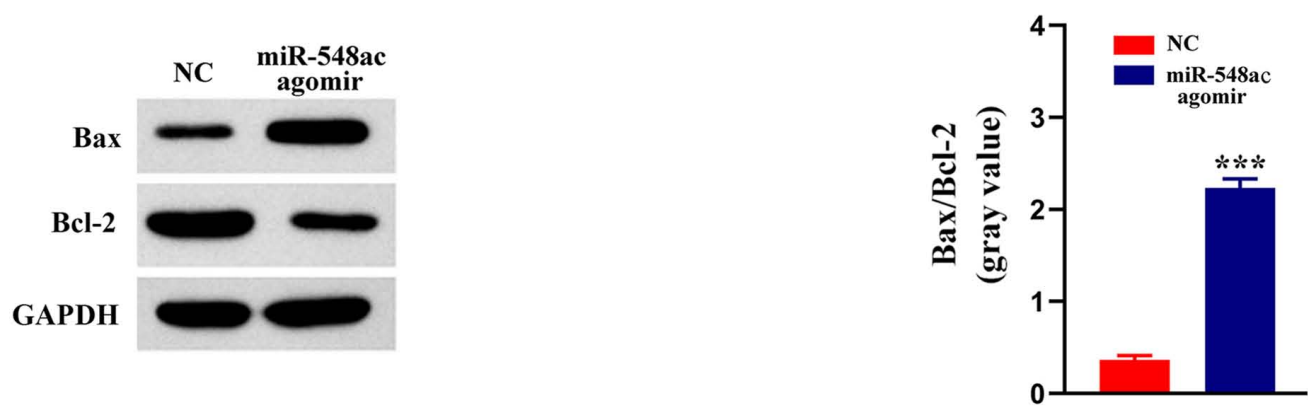

Figure 4. miR-548ac affects viability, formation of colonies, cell cycle distribution and apoptosis of laryngeal cancer cells. (A) MTT assay was performed to assess cell viability in LSC-1 cells transfected with miR-548ac antagomir or NC. ${ }^{* * *} \mathrm{P}<0.001$, miR-548ac agomir vs. NC. (B) Effect of miR-548ac on colony formation of laryngeal cancer cells. Representative micrographs (left) and relative quantification analysis of colonies (right). ${ }^{* *} \mathrm{P}<0.01$, miR-548ac agomir vs. NC. (C) Flow cytometric analysis of apoptosis. ${ }^{* *} \mathrm{P}<0.01$, miR-548ac agomir vs. NC. (D) Flow cytometric analysis of cell cycle distribution. Histograms depict the proportion of LSC-1 cells in the G1, S and G2 phases. ${ }^{* * *} \mathrm{P}<0.001$, miR-548ac agomir $\left(\mathrm{G}_{1}\right)$ vs. NC $\left(\mathrm{G}_{1}\right) ;{ }^{* *} \mathrm{P}<0.01$, miR-548ac agomir $\left(\mathrm{G}_{2}\right)$ vs. NC $\left(\mathrm{G}_{2}\right)$. $(\mathrm{E})$ Western blot analysis was performed to assess protein expression levels of Bax and Bcl-2. Histograms depict the gray value of Bax/Bcl-2. ${ }^{* * *} \mathrm{P}<0.001$, miR-548ac agomir vs. NC. miR, microRNA; LSC-1, laryngeal squamous cell carcinoma; NC, negative control; Bcl-2, B-cell lymphoma 2; PI, propidium iodide.

$\mathrm{P}<0.0001$; Fig. 4A). The results of the colony formation assay demonstrated that the number of cell colonies was significantly lower in the experimental group compared with the control group $(65.44 \pm 4.34 \%$ of the control; $\mathrm{P}=0.0094$; Fig. $4 \mathrm{~B})$. The results of the Annexin V-PI assay demonstrated that the apoptotic rate was significantly higher in the experimental compared with the control group (7.27 \pm 1.22 -fold of the control; $\mathrm{P}=0.003$;
Fig. 4C). The cell cycle distribution assay demonstrated a higher proportion of cells transfected with miR-548ac agomir in the $\mathrm{G}_{1}$ phase compared with the control group (Fig. 4D). The protein expression levels of Bax and Bcl-2 were detected via western blot analysis, and the gray value was used for relative quantification. The western blots showed that Bcl-2 protein expression was higher and Bax protein expression was lower in 
A

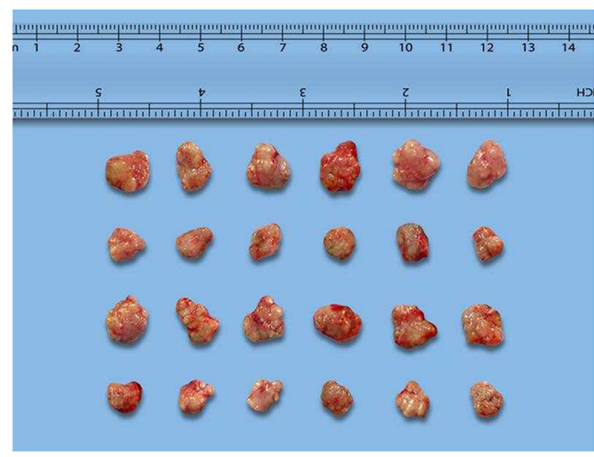

Blank vector

ShTMEM158

miR-548ac NC

miR-548ac agomir

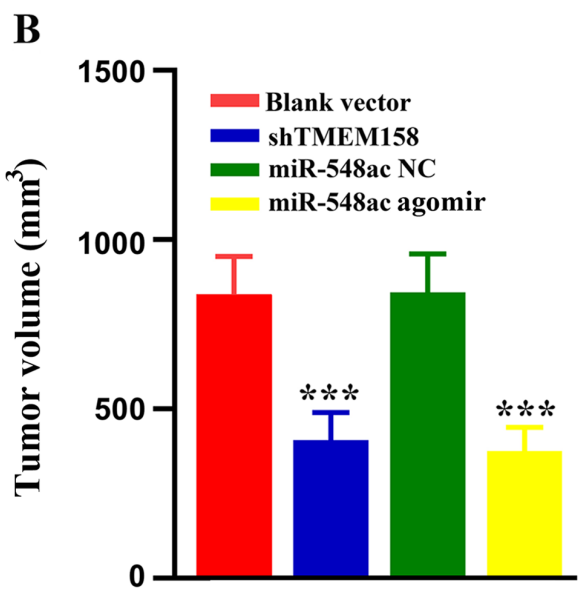

C

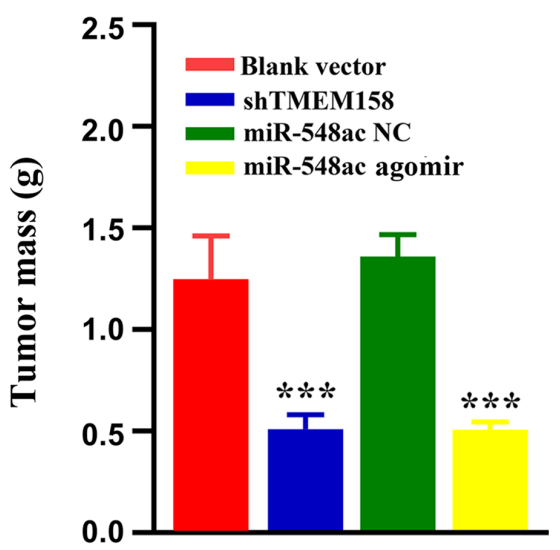

Figure 5. Effects of TMEM158 and miR-548ac on tumor growth in vivo. (A) Representative images of tumors derived from nude mice in the blank vector, shTMEM158, miR-548ac NC and miR-548ac agomir groups. (B) Tumor volumes in the different groups. (C) Tumor masses in the different groups. ${ }^{* * *} \mathrm{P}<0.001$ vs. blank vector group and miR-548ac NC group. TMEM158, transmembrane protein 158; miR, microRNA; sh, short hairpin; NC, negative control.

$\mathrm{NC}$ group compared with the experimental group. Gray value ratio of $\mathrm{Bax}$ and $\mathrm{Bcl}-2$ was significantly higher in the experimental group compared with the control group $(6.19 \pm 0.46$ fold of the control; $\mathrm{P}=0.00045$; Fig. 4E). Collectively, these results indicate that overexpressing miR-548ac significantly inhibits the viability of laryngeal cancer cells. Thus, it is speculated that miR-548ac plays a cancer suppressive role in laryngeal cancer.

Effect of TMEM158 and miR-548ac on tumor growth in vivo. A xenograft model was used to determine the effects of TMEM158 and miR-548ac on tumor growth in vivo. As presented in Fig. 5A, the tumors were smaller in the shTMEM158 and miR-548ac agomir groups compared with the corresponding control groups. The tumor volume in the shTMEM158 group was $49.05 \pm 9.78 \%$ that of the blank vector group $(\mathrm{P}<0.001)$, while the tumor volume in the miR-548ac group was $45.36 \pm 10.54 \%$ that of the miR-548ac NC group $(\mathrm{P}<0.001)$ (Fig. 5B). The tumor mass in the shTMEM158 group was $42.21 \pm 11.47 \%$ that of the blank vector group $(\mathrm{P}<0.0001)$, while the tumor mass in the miR-548ac group was $37.49 \pm 4.43 \%$ that of the miR-548ac NC group $(\mathrm{P}<0.001)$ (Fig. 5C).

\section{Discussion}

miRNAs function in repressing translation of target mRNAs or degrading it, thus negatively regulating the expression of target genes following transcription (18). The regulatory mechanism of miRNAs to their target genes has been extensively studied and demonstrated to be involved in biological events, such as cell proliferation, apoptosis, differentiation, exercise and angiogenesis $(19,20)$. It has been reported that the miR-548 family can inhibit the growth of several tumors. For example, Shi et al (15) reported that mir-548-3p inhibits the proliferation of breast cancer cells by regulating ECHS1 expression, suggesting its potential as a therapeutic target for breast cancer. Furthermore, Wang et al (14) demonstrated that mir-548-3p can effectively inhibit the proliferation of lung cancer cells by inhibiting the PI3K/Akt signaling pathway, indicating that miR-548b-3p has the potential to be developed as an antitumor target of lung cancer. The results of the present study demonstrated that LSC-1 cell viability significantly decreased following overexpression of miR-548ac, whereby some cells exhibited early apoptosis accompanied by activation of the apoptosis factor, Bax, and further cell cycle arrest at the $\mathrm{G}_{1}$ phase. Thus, it was speculated that overexpression of miR-548ac in laryngeal cancer cells significantly inhibits cell proliferation and induces apoptosis. Collectively, the results of the present study suggest that miR-548ac may function as a tumor suppressor in laryngeal cancer.

The expression and molecular mechanism of TMEM158 have been reported in different types of tumor. For example, Iglesias et al (21) suggested that TMEM158 acts as an oncogene in the development of colon cancer. In addition, Mohamed et al (8) demonstrated that TMEM158 interference significantly decreases the toxic effect exerted by cisplatin 
on NSCLC cells, suggesting that TMEM158 is associated with the sensitivity of chemotherapy drugs. Zirn et al (22) reported that TMEM158 is highly expressed in CTNNB1 mutant nephroblastoma, which may be associated with the Ras and Wnt signaling pathways. Furthermore, Liu et al (23) demonstrated that TMEM158 is a key regulatory factor of colorectal carcinogenesis and drug resistance, and thus may be a promising target for colorectal cancer treatment. The results of the present study demonstrated that TMEM158 was highly expressed in laryngeal carcinoma tissues from the TCGA database, which was verified via cell line experiments. The results demonstrated that LSC- 1 cell viability significantly decreased following TMEM158 silencing, which induced early apoptosis, accompanied by activation of the apoptotic factors (Bax). Simultaneously, TMEM158 silencing resulted in cell arrest in the $G_{1}$ phase. Collectively, these results suggest that TMEM158 may act as an oncogene, affecting the viability of laryngeal cancer cells.

miRNAs regulate the expression of $\sim 30 \%$ of human protein-coding genes. Each miRNA can regulate hundreds of mRNA genes, and the same target gene can be regulated by multiple miRNAs, to regulate the expression of thousands of proteins directly or indirectly (24). miRNAs regulate gene expression by specifically pairing with the 3'-UTR region of their target gene, resulting in the degradation of the target mRNA or post transcriptional silencing, thus inhibiting protein synthesis $(25,26)$. The results of the present study demonstrated that TMEM158 mRNA and protein expression levels significantly decreased following overexpression of miR-548ac in LSC-1 cells. The results of the dual-luciferase reporter assay confirmed that miR-548ac directly binds to the 3'-UTR of TMEM158, thus inhibiting TMEM158 expression. Consistent with previous findings, the results of the present study demonstrated that miR-548ac induces apoptosis in LSC-1 cells by targeting TMEM158.

The present results suggest that miR-548ac functions as a crucial cancer suppressor in laryngeal cancer, which induces apoptosis in laryngeal cancer cells by suppressing TMEM158. The present findings enrich the pathogenesis of laryngeal cancer and provide a new target and new insights for the treatment of laryngeal cancer. The limitation of this study is that there is no biological detection of TMEM158 and miR-548ac in clinical samples. At the same time, there is no detection on the influence of the follow-up signal pathway. These limitations will be addressed in future studies.

\section{Acknowledgements}

The authors would like to thank The Cancer Genome Atlas (TCGA) project for providing the data.

\section{Funding}

Support for the study was provided by the Tianjin Natural Science Foundation (No 20170842).

\section{Availability of data and materials}

All data generated or analyzed during this study are included in this published article.

\section{Authors' contributions}

FS designed the experiment and wrote the manuscript; YY contributed to animal and cell experiments; JL performed the statistical and bioinformatics analysis. All authors read and approved the final manuscript.

\section{Ethics approval and consent to participate}

The present study was approved by the Ethics Committee of Tianjin Union Medical Center (Tianjin, China; approval no. 2018-269,). All animal experiments were performed in accordance with the National Institutes of Health Guide for the Care and Use of Laboratory Animals (NIH Publications no. 8023 , revised in 1978).

\section{Patients consent for publication}

Not applicable.

\section{Competing interests}

The authors declare that they have no competing interests.

\section{References}

1. Beibei Y, Rong Y, Yunfei Y and Wenchao Z: Research progress regarding surgical margins, molecular margins, and prognosis of laryngeal carcinoma. Ear Nose Throat J 145561320903146, 2020 (Epubahead of print)

2. Olaleye O, Siddiq S, Ekrikpo U and Kazi R: Regional differences in incidence and mortality trends in cancers of the larynx, thyroid, oral cavity and pharynx in England and Scotland: 1975-2002. Open Epidemiol J 3: 70-78, 2013.

3. Bray F, Ferlay J, Soerjomataram I, Siegel RL, Torre LA and Jemal A: Global cancer statistics 2018: GLOBOCAN estimates of incidence and mortality worldwide for 36 cancers in 185 countries. CA Cancer J Clin 68: 394-424, 2018.

4. Steuer CE, El-Deiry M, Parks JR, Higgins KA and Saba NF: An update on larynx cancer. CA Cancer J Clin 67: 31-50, 2017.

5. Menicagli R, Bolla G, Menicagli L and Esseridou A: The possible role of diabetes in the etiology of laryngeal cancer. Gulf J Oncolog 1: 44-51, 2017.

6. Barradas M, Gonos ES, Zebedee Z, Kolettas E, Petropoulou C, Delgado MD, Leon J, Hara E and Serrano M: Identification of a candidate tumor-suppressor gene specifically activated during Ras-induced senescence. Exp Cell Res 273: 127-137, 2002.

7. Cheng Z, Guo J, Chen L, Luo N, Yang W and Qu X: Overexpression of TMEM158 contributes to ovarian carcinogenesis. J Exp Clin Cancer Res 34: 75, 2015.

8. Mohammed Ael S, Eguchi H, Wada S, Koyama N, Shimizu M, Otani K, Ohtaki M, Tanimoto K, Hiyama K, Gaber MS and Nishiyama M: TMEM158 and FBLP1 as novel marker genes of cisplatin sensitivity in non-small cell lung cancer cells. Exp Lung Res 38: 463-474, 2012.

9. Fu Y, Yao N, Ding D, Zhang X, Liu H, Ma L, Shi W, Zhu C and Tang L: TMEM158 promotes pancreatic cancer aggressiveness by activation of TGF $\beta 1$ and PI3K/AKT signaling pathway. J Cell Physiol 235: 2761-2775, 2020.

10. Hummel R, Maurer J and Haier J: MicroRNAs in brain tumors: A new diagnostic and therapeutic perspective? Mol Neurobiol 44: 223-234, 2011

11. Rouleau S, Glouzon JS, Brumwell A, Bisaillon $M$ and Perreault JP: 3' UTR G-quadruplexes regulate miRNA binding. RNA 23: 1172-1179, 2017.

12. Xiao X, Cao Y and Chen H: Profiling and characterization of microRNAs responding to sodium butyrate treatment in A549 cells. J Cell Biochem 119: 3563-3573, 2018.

13. Liang T, Guo L and Liu C: Genome-wide analysis of mir-548 gene family reveals evolutionary and functional implications. J Biomed Biotechnol 2012: 679563, 2012. 
14. Wang Z, Wu X, Hou X, Zhao W, Yang C, Wan W and Chen L: MiR-548b-3p functions as a tumor suppressor in lung cancer. Lasers Med Sci 35: 833-839, 2020

15. Shi Y, Qiu M, Wu Y and Hai L: MiR-548-3p functions as an anti-oncogenic regulator in breast cancer. Biomed Pharmacother 75: 111-116, 2015.

16. Schmittgen TD and Livak KJ: Analyzing real-time PCR data by the comparative C(T) method. Nat Protoc 3: 1101-1108, 2008.

17. Tashiro K, Kuroki N, Einama T, Iwasaki T, Miyata Y, Aosasa S, Inoue Y, Takahashi Y, Ogata S, Ueno H, et al: Prognostic significance of regional lymph node metastasis according to station in ampullary carcinoma. J Hepatobiliary Pancreat Sci 2020 (Epub ahead of print).

18. Bartel DP: MicroRNAs: Target recognition and regulatory functions. Cell 136: 215-233, 2009.

19. Mellis D and Caporali A: MicroRNA-based therapeutics in cardiovascular disease: Screening and delivery to the target. Biochem Soc Trans 46: 11-21, 2018.

20. Qadir MI and Faheem A: MiRNA: A diagnostic and therapeutic tool for pancreatic cancer. Crit Rev Eukaryot Gene Expr 27: 197-204, 2017.

21. Iglesias D, Fernandez-Peralta AM, Nejda N, Daimiel L, Azcoita MM, Oliart S and Gonzalez-Aguilera JJ: RIS1, a gene with trinucleotide repeats, is a target in the mutator pathway of colorectal carcinogenesis. Cancer Genet Cytogenet 167: 138-144, 2006.
22. Zirn B, Samans B, Wittmann S, Pietsch T, Leuschner I, Graf N and Gessler M: Target genes of the WNT/beta-catenin pathway in Wilms tumors. Genes Chromosomes Cancer 45: 565-574, 2006.

23. Liu L, Zhang J, Li S, Yin L and Tai J: Silencing of TMEM158 inhibits tumorigenesis and multidrug resistance in colorectal cancer. Nutr Cancer 72: 662-671, 2020.

24. Kura B, Kalocayova B, LeBaron TW, Frimmel K, Buday J, Surovy J and Slezak J: Regulation of microRNAs by molecular hydrogen contributes to the prevention of radiation-induced damage in the rat myocardium. Mol Cell Biochem 457: 61-72, 2019.

25. Fabian MR, Sonenberg N and Filipowicz W: Regulation of mRNA translation and stability by microRNAs. Annu Rev Biochem 79: 351-379, 2010.

26. Correia de Sousa M, Gjorgjieva M, Dolicka D, Sobolewski C and Foti M: Deciphering miRNAs' Action through miRNA Editing. Int J Mol Sci 20: 6249, 2019.

(i) (9) This work is licensed under a Creative Commons Attribution-NonCommercial-NoDerivatives 4.0 International (CC BY-NC-ND 4.0) License. 